\title{
Inflammatory myofibroblastic tumor of the larynx: Case report
}

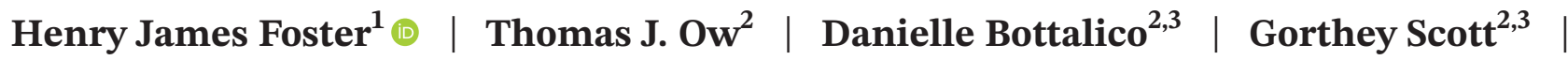 \\ Berrin Ustun ${ }^{4}$ Keivan Shifteh ${ }^{2,3}$
}

\author{
${ }^{1}$ Albert Einstein College of Medicine, \\ Bronx, New York, USA \\ ${ }^{2}$ Department of Otorhinolaryngology- \\ Head and Neck Surgery, Montefiore \\ Medical Center/Albert Einstein College \\ of Medicine, Bronx, New York, USA \\ ${ }^{3}$ Department of Radiology, Montefiore \\ Medical Center/Albert Einstein College \\ of Medicine, Bronx, New York, USA \\ ${ }^{4}$ Department of Pathology, Montefiore \\ Medical Center/Albert Einstein College \\ of Medicine, Bronx, New York, USA

\section{Correspondence} \\ Keivan Shifteh, Albert Einstein College \\ of Medicine/Montefiore Medical \\ Center, 111 E 210th Street Bronx, NY \\ 10467, USA. \\ Email: Kshifteh@montefiore.org \\ Funding information \\ No funding was used in this report
}

\begin{abstract}
Inflammatory myofibroblastic tumor is usually a benign tumor of mesenchymal origin that is rarely found in the larynx. This case explores the unique laryngeal location and presentation of this tumor as well as the challenging radiographic and histologic findings.
\end{abstract}

\section{K E Y W O R D S}

benign lesions, chemotherapy, larynx, microlaryngeal surgery, radiology

\section{1 | INTRODUCTION}

Inflammatory myofibroblastic tumor (IMT) of the larynx is rare and has links to smoking and viral/bacterial exposure. ${ }^{1-4}$ Nonspecific symptoms are common as it is typically a painless mass. ${ }^{1}$ IMT has a variable radiographic appearance, but has characteristic histopathology findings. Complete surgical excision offers a low rate of recurrence.

\section{2 | CASE PRESENTATION}

A 41-year-old female patient with a history of asthma and gastroesophageal reflux presented to the emergency department with 2-3 days of a choking sensation and shortness of breath. She also reported 4 months of voice changes and aspiration of thin liquids. There was no tobacco or alcohol use history. At presentation, the patient had stridor, and fiberoptic laryngoscopy revealed a large pink ball-valving polypoid lesion pedicled on the glottis anteriorly and obstructing $75 \%$ of the airway. Oxygen saturation levels were normal. Pre and postcontrast computed tomography (CT) of the neck revealed an approximately well-defined, partially enhancing $1.5-\mathrm{cm}$ soft tissue mass arising from the anterior commissure of the larynx with moderate compromise of the laryngeal lumen (Figure 1AC). The patient was admitted to a step-down unit for continuous respiratory and pulse oximetry monitoring. 
The next day, the patient was taken to the operating theater by the otolaryngology service. General anesthesia was induced, and the patient was quickly and carefully intubated under direct laryngoscopy. The mass was resected using cold microlaryngeal techniques. The mass was found to be broadly attached along the anterior commissure of the larynx (Figure 1D). Frozen section biopsies suggested a neoplasm, but the lesion could not be further characterized. The mass was fully excised up to the lesion's anterior glottic and subglottic attachments. Care was taken to avoid significant trauma to the true vocal cord mucosa and glottic ligaments. Final pathology characterization showed the specimen was positive for anaplastic lymphoma kinase (ALK) and SMA and was negative for cytokeratin, desmin, MSA, nuclear myogenin, nuclear myoD1, S100, CD34, p63, p40, and CK903. Mitotic activity was present without atypical mitosis (Figure 2).

Follow-up contrast-enhanced $\mathrm{CT}$ at 2 and 6 months (Figure 3A, B) revealed no evidence of recurrence. The patient returned to the operating room 8 months after initial presentation for a direct follow-up evaluation. No evidence of recurrent disease was found, but a small anterior laryngeal web was identified (Figure 3C).

\section{DISCUSSION}

IMT is a benign and uncommon mesenchymal tumor. ${ }^{1,2}$ IMT occurs most commonly in the lungs, gastrointestinal tract, and liver, but rarely arises in the larynx. ${ }^{1-3}$ Laryngeal IMT has been reported most commonly in the vocal folds, although a few cases in the aryepiglottic folds have been reported. ${ }^{4}$ Lesions are usually $0.4-3.5 \mathrm{~cm}$ in size. ${ }^{3,4}$ IMT primarily affects children and younger adults (mean age of 43)..$^{1-4}$

IMT originates from an inflammatory response and is related to smoking, immune responses, and trauma. ${ }^{1-4}$ IMT has also been linked to infectious agents including Epstein-Barr virus, human immunodeficiency virus, human herpesvirus-8, and bacteria such as Escherichia coli and Nocardia. ${ }^{1,4}$

Clinical presentation with IMT is often nonspecific. Constitutional symptoms including weight loss, fever, and fatigue occur in 15\%-30\% of patients. IMTs are usually painless, but may present with swelling, induration, or obstructive symptoms and/or voice changes. ${ }^{1-3}$ Laboratory values can show microcytic anemia, elevated erythrocyte sedimentation rate (ESR), thrombocytosis, and polyclonal hypergammaglobulinemia. ${ }^{2,3}$ These laboratory findings have not been demonstrated in laryngeal IMT, and were not found in this current case although an ESR was not measured. ${ }^{2,3}$

IMT has a variable radiographic appearance that can be nonspecific, suggestive of an infiltrative/malignant lesion, or granulomatous disease. ${ }^{1}$ It ranges from an illdefined infiltrating lesion to a well-circumscribed mass with inflammatory and fibrotic regions. ${ }^{2}$ Fibrotic sections display delayed and persistent contrast enhancement, but attenuation on CT is variable as is echogenicity on ultrasound. ${ }^{2}$ Laryngeal IMT has been reported on CT showing uneven enhancement or as a subglottic mass not invading the laryngeal skeleton. ${ }^{3}$ Laryngeal IMT has not been well described on magnetic resonance imaging (MRI), but any fibrosis will produce a low signal intensity region. ${ }^{2}$ Additionally, in one case, fat-saturated $\mathrm{T} 1$ gradient-echo images showed a mild spontaneously hyperintense vocal fold, and on fat-saturated T2-weighted images showed relative hyperintensity. ${ }^{3}$ Strong contrast enhancement was also present. ${ }^{3}$

Histologically, IMT demonstrates myofibroblastic proliferating spindle cells with infiltrating plasma cells, lymphocytes, and eosinophils. ${ }^{3}$ Other patterns include compact fascicular spindle cells with myxoid and collagenized regions with an inflammatory cell background, and a denser collagen pattern with plate-like collagen and less cellularity. ${ }^{4}$ Immunochemistry staining of IMT shows reactivity to vimentin, SMA, muscle-specific actin, and desmin while being negative for myoglobin and S100. ${ }^{1,3}$ Additionally, ALK-1 gene translocations
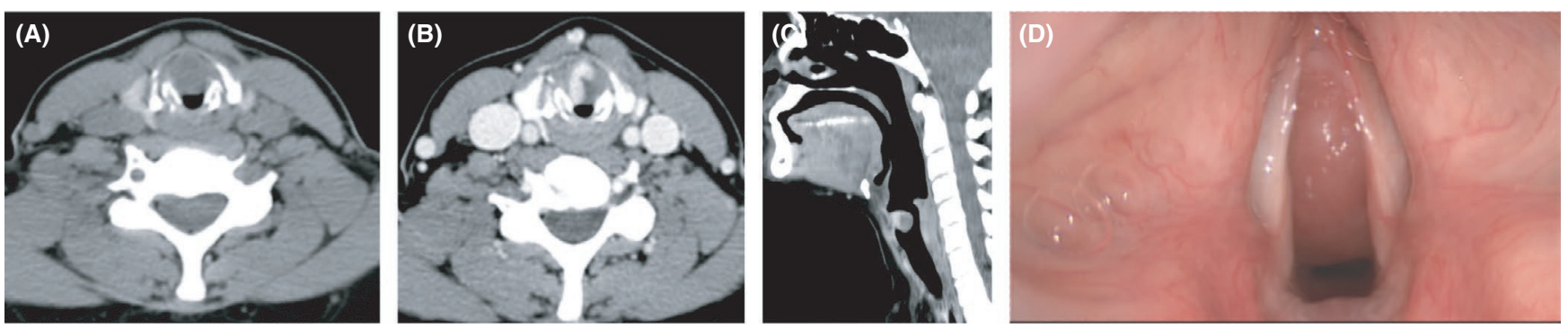

F I G U RE 1 (A) Axial noncontrast CT examination of the neck at the level of the larynx demonstrates a well-defined polypoid soft tissue mass arising from the anterior commissure. Axial (B) and sagittal (C) contrast-enhanced CT examination of the neck demonstrates partial enhancement. Intraoperative direct laryngoscopy (D) showing the 1.5-cm mass attached to the anterior commissure 


\section{FI G URE 2 (A) Exophytic} proliferation of spindle cells in a loose myxoid background (H\&E, 40×). (B) Spindle cells admixed with chronic inflammatory cells in a myxoid background (H\&E, 200×). (C) Tumor cells have low nuclear cytoplasmic ratios, hypochromatic nuclei with open chromatin and prominent nucleoli. Mitotic figures are present (H\&E, 400×). (D) Tumor cells show positive staining for ALK-1 by immunohistochemistry (ALK-1, 200×)
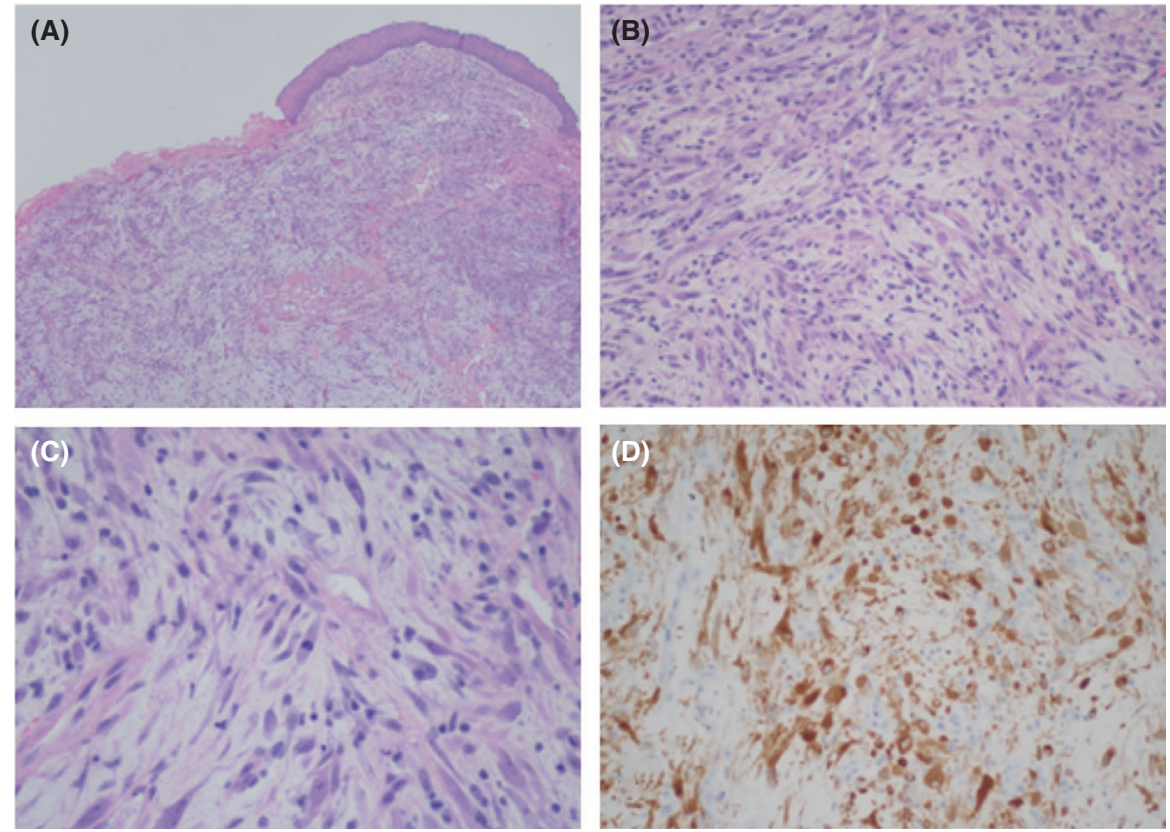
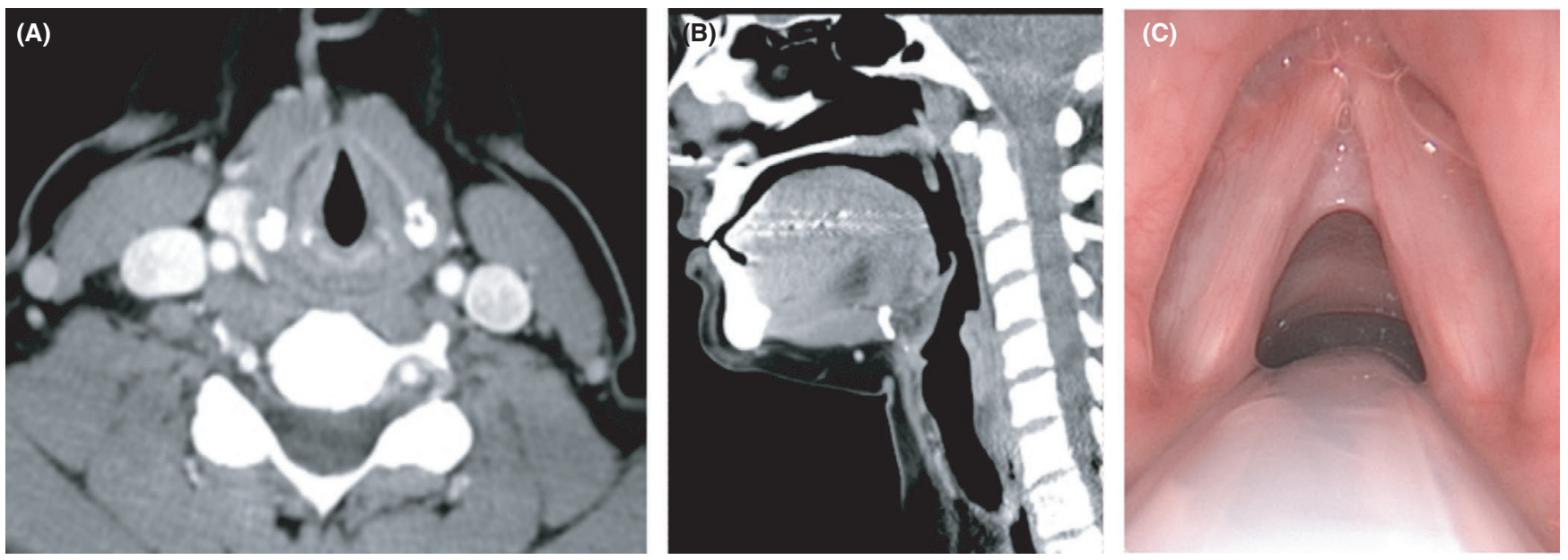

F I G U RE 3 Axial (A) and sagittal (B) contrast-enhanced CT examination of the neck after 6 months follow-up demonstrates no evidence of recurrent disease. Intraoperative direct laryngoscopy (C) showing no recurrence of disease and only a small anterior laryngeal web

are present in about half of IMTs resulting in overexpression of ALK. Fusion partners have been identified including tropomyosin, clathrin heavy chain, and Ranbinding protein $2 .{ }^{1,2}$ ALK-1 positivity is relatively specific for IMT, and is frequent in laryngeal IMT, although bears no morphologic correlation. ${ }^{1,4}$ IMTs without ALK-1 translocations still show the presence of other fusion proteins involving platelet-derived growth factor, ROS1, tropomyosin 3 and 4, cysteinyl tRNA synthetase, and/or Ran-binding protein.,

Squamous cell carcinoma, papilloma, and leiomyosarcoma were considered in the differential, but the histopathology findings supported IMT.
The leading treatment for laryngeal IMT is surgical excision, which offers a low recurrence rate and good prognosis. ${ }^{2-4}$ Chemotherapy or radiotherapy is considered when morbidity of surgery or patient comorbid conditions preclude surgery. ${ }^{2,3}$

The ALK inhibitor crizotinib is a novel treatment for ALK + IMT. Crizotinib therapy has shown complete or partial remission in cases where only partial tumor resection was possible. ${ }^{5}$ In four cases, crizotinib therapy resulted in remission without surgical intervention. ${ }^{5}$ However, these data are limited by publication bias, complex treatment regimens, and variable follow-up time, thus, further study is needed. ${ }^{5}$ 


\section{CONCLUSION}

Here, we report a case of laryngeal IMT that presented as a laryngeal mass causing airway obstruction. The lesion was confirmed histologically after definitive treatment with microlaryngeal excision. Although rare, this tumor can be considered in a differential diagnosis of benign laryngeal masses. Diagnosing laryngeal IMT may lead to improved clinical care given the favored prognosis with complete surgical removal or newer options for lesions that preclude complete excision.

\section{ACKNOWLEDGEMENTS}

There are no acknowledgments.

\section{CONFLICT OF INTEREST}

There are no conflicts of interest.

\section{AUTHOR CONTRIBUTIONS}

HF drafted the primary manuscript. TJ, DB, SG, BU, and KS edited the manuscript. TJ, DB, and SG directly involved in the clinic with patient/case/operation. BU directly involved with the histology/pathology images. BU and KS discussed the manuscript. KS is the corresponding author and directly involved with the radiology and imaging in the case and in the radiographic images/captions.

\section{CONSENT}

Written informed consent was provided by the patient for publication of this case and the images.

\section{DATA AVAILABILITY STATEMENT}

The data that support the findings of this study are available from the corresponding author upon reasonable request.

\section{ORCID}

Henry James Foster (1) https://orcid.

org/0000-0002-5404-4899

\section{REFERENCES}

1. Palaskar S, Koshti S, Maralingannavar M, Bartake A. Inflammatory myofibroblastic tumor. Contemp Clin Dent. 2011;2(4):274-277.

2. Surabhi VR, Chua S, Patel RP, Takahashi N, Lalwani N, Prasad SR. Inflammatory myofibroblastic tumors: current update. Radiol Clin North Am. 2016;54(3):553-563. https:// doi.org/10.1016/j.rcl.2015.12.005. Epub 2016 Mar 12 PMID: 27153788.

3. Do BA, Varshney R, Zawawi F, Levental M, Caglar D, Young J. Inflammatory myofibroblastic tumor of the larynx-a case report. J Voice. 2014;28(2):258-261. https://doi.org/10.1016/j.jvoice.2013.09.004. Epub 2013 Dec 8 PMID: 24321588.

4. Ni C, Xu YY, Zhou SH, Wang SQ. Differential diagnosis of inflammatory myofibroblastic tumour and low-grade myofibroblastic sarcoma: two case reports with a literature review. J Int Med Res. 2011;39(1):311-320. https://doi.org/10.1177/14732 3001103900134. PMID: 21672335.

5. Theilen TM, Soerensen J, Bochennek K, et al. Crizotinib in ALK+ inflammatory myofibroblastic tumors-Current experience and future perspectives. Pediatr Blood Cancer. 2018;65(4). https://doi.org/10.1002/pbc.26920. Epub 2017 Dec 29. PMID: 29286567.

How to cite this article: Foster HJ, Ow TJ, Bottalico D, Scott G, Ustun B, Shifteh K. Inflammatory myofibroblastic tumor of the larynx: Case report. Clin Case Rep. 2021;9:e04796. https:// doi.org/10.1002/ccr3.4796 